\title{
Characterization and Functionality of Immidazolium Ionic Liquids Modified Magnetic Nanoparticles
}

\author{
Ying Li, ${ }^{1,2}$ Ning Tang, ${ }^{1,3}$ Fuyuhiko Inagaki, ${ }^{1}$ Chisato Mukai, ${ }^{1}$ and Kazuichi Hayakawa ${ }^{1}$ \\ ${ }^{1}$ Institute of Medical, Pharmaceutical and Health Sciences, Kanazawa University, Kakuma-machi, Kanazawa 920-1192, Japan \\ ${ }^{2}$ Department of Chemical and Chemical Engineering, Henan Institute of Science and Technology, Xinxiang, Henan 453003, China \\ ${ }^{3}$ Hyogo College of Medicine, 1-1 Mukogawa-cho, Nishinomiya, Hyogo 663-8501, Japan
}

Correspondence should be addressed to Ying Li; rklxy@yahoo.com.cn and Kazuichi Hayakawa; hayakawa@kanazawa-u.ac.jp

Received 12 January 2013; Revised 13 March 2013; Accepted 31 March 2013

Academic Editor: Chinnappan Baskar

Copyright (C) 2013 Ying Li et al. This is an open access article distributed under the Creative Commons Attribution License, which permits unrestricted use, distribution, and reproduction in any medium, provided the original work is properly cited.

1,3-Dialkylimidazolium-based ionic liquids were chemically synthesized and bonded on the surface of magnetic nanoparticles (MNPs) with easy one-step reaction. The obtained six kinds of ionic liquid modified MNPs were characterized with transmission electron microscopy, thermogravimetric analysis, magnetization, and FTIR, which owned the high adsorption capacity due to the nanometer size and high-density modification with ionic liquids. Functionality of MNPs with ionic liquids greatly influenced the solubility of the MNPs with organic solvents depending on the alkyl chain length and the anions of the ionic liquids. Moreover, the obtained MNPs showed the specific extraction efficiency to organic pollutant, polycyclic aromatic hydrocarbons, while superparamagnetic property of the MNPs facilitated the convenient separation of MNPs from the bulks water samples.

\section{Introduction}

Magnetic nanoparticles (MNPs) have potential application for material science and biomedicine because of their unique superparamagnetism and large surface area. MNPs can be functionalized by modifying their surfaces with various materials to achieve the special purposes [1-4]. Coated shellcore MNPs with carbon, alumina, and surfactants have been recently used to preconcentrate inorganic/organic contaminants in environmental and biological samples [5-8]. Ionic liquids, which can be tuned by choosing of the cations and anions independently, exhibited unique physicochemical properties, such as tunable miscibility with water and organic solvents, and have potential applications as electrolyte materials, green solvents, catalysts, and separation mediums [914]. $\mathrm{N}, \mathrm{N}^{\prime}$-diallylimidazolium-based ionic liquids have also been used as extractant to highly enrich organic pollutants from aqueous samples in microextraction techniques, such as dispersed liquid-liquid microextraction and single drop microextraction, or to functionalize the stationary phase of chromatographic column and solid fiber membranes [15-21]. MNPs coated with ionic liquid were applied to preconcentrate polycyclic aromatic hydrocarbons (PAHs) in water samples through forming mixed hemimicelles on the surface of MNPs. However, it has been noted that the coating of ionic liquid is noncovalent, which means that ionic liquid is more easily desorbed from surface and preconcentration of organic pollutants is strongly affected by the extraction parameters. In this work, a novel kind of modified MNPs with $\mathrm{N}, \mathrm{N}^{\prime}$ dialkylimidazolium-based ionic liquids containing different alkyl chain lengths and inorganic anions were synthesized by covalent bonding. The functionalization of the resultant MNPs were confirmed by transmission electron microscopy (TEM), thermogravimetric analysis (TGA), and Fourier transform infrared spectra (FTIR). Although the naked and modified MNPs had similar saturation magnetizations, the obvious differences of solubility in water and organic solvents were observed. The modified MNPs were used to preconcentrate the trace level of carcinogenic PAHs in environmental water samples. To the best of our knowledge, this is the first 
work about application of the chemically bonded ionic liquid modified MNPs for detecting carcinogenic organic pollutants in environmental water samples.

\section{Experimental}

3-(2-Imidazolin-1-yl) propyltriethoxysilane was purchased from Sigma-Aldrich. 1-Chlorohexane, 1-chlorooctane, and 1chlorodecane were purchased from Tokyo Chemical Industry Co., Ltd. (Japan). $\mathrm{NaPF}_{6}, \mathrm{FeCl}_{3} \cdot 6 \mathrm{H}_{2} \mathrm{O}$, and $\mathrm{FeCl}_{2} \cdot 4 \mathrm{H}_{2} \mathrm{O}$ were purchased from Wako Pure Chemical Industries, Ltd. PAHs standards were purchased from Supelco (USA). Organic solvents are HPLC grade. A Milli-Q water purification system (USA) was used to prepare ultrapure water.

The magnetic iron oxide nanoparticles (MNPs) were synthesized by the Massart's method with minor changes (Scheme 1) [22]. Six kinds of (3-triethoxysilylpropyl)-4,5-di hydroimidazolium (TIM) ionic liquids, (1-hexyl-3-TIM chlo ride (HTIMCl), 1-octyl-3-TIM chloride (OTIMCl), 1-decyl3-TIM chloride (DTIMCl), 1-hexyl-3-TIM hexafluorophosphate $\left(\mathrm{HTIMPF}_{6}\right)$, 1-octyl-3-TIM hexafluorophosphate (OT$\mathrm{IMPF}_{6}$ ), and 1-decyl-3-TIM hexa fluorophosphate (DTI$\left.\mathrm{MPF}_{6}\right)$ ) were synthesized (Table 1). The synthesis method was as follows (Scheme 1): a mixture of 3-(2-imidazolin-1yl) propyltriethoxysilane and 1-chloroalkane was stirred at $120^{\circ} \mathrm{C}$ for $8 \mathrm{~h}$. Then the mixture was cooled to room temperature to afford 1-alkyl-3-(3-triethoxysilylpropyl)-4,5-dihydroimidazolium chloride as an orange oil. To a solution of 1-alkyl3-(3-triethoxysilylpropyl)-4,5-dihydroimidazolium chloride in $\mathrm{CH}_{3} \mathrm{CN}$ was added $\mathrm{NaPF}_{6}$ at room temperature. After stirring for $5 \mathrm{~min}$, the reaction mixture was filtrated, and the volatile components were evaporated off to afford 1-alkyl3-(3-triethoxysilylpropyl)-4,5-dihydroimidazolium hexafluorophosphate. The resultants were colourless powders when alkyl were hexyl and decyl with melting point of $131-132^{\circ} \mathrm{C}$ and $123-124^{\circ} \mathrm{C}$, respectively, while the resultant was an orange oil when the alkyl was octyl. The obtained ionic liquids were confirmed by FTIR, H-NMR, and C-NMR. The ionic liquid-functional MNPs were obtained through adding a solution of the desired functionalized ionic liquid and $28 \%$ aqueous $\mathrm{NH}_{3}$. After mechanical stirring for $36 \mathrm{~h}$ at room temperature, the ionic liquid-functionalized MNPs were magnetically separated, washed with EtOH, and concentrated to dryness under reduced pressure (less than $1 \mathrm{mmHg}$ ) to give the desired product as black powders. The ionic liquid modified MNPs are designated as MNP-X, where X is the abbreviation of the ionic liquid (Table 1).

Morphologies were observed by TEM (JEM-2010, Tokyo, Japan) and HRTEM images were obtained using the same transmission electron microscope. Thermogravimetric analysis (TGA) was done with a TG/DTA 6200 (SII Nano Technology, Tokyo, Japan). X-ray powder diffraction (XRD) was carried out on a Rigaku X-ray RINT2200 (Ultima, Tokyo, Japan) diffractometer with $\mathrm{Cu} \mathrm{K}_{\alpha}$ radiation $(\lambda=0.1542 \mathrm{~nm})$. Magnetic properties were measured with a SQUID magnetometer (Quantum Design MPMS). FTIR were taken in $\mathrm{KBr}$ pressed pellets with a suitable ratio of MNP to $\mathrm{KBr}$ on an FTIR-8700 (Shimadzu, Kyoto, Japan).
The functional MNPs were added to the filtered river water sample to enrich the organic pollutants, polycyclic aromatic hydrocarbons (PAHs), under the sonication effect. After that an NdFeB magnet was then utilized to collect the MNPs from samples, and then the enriched PAHs were eluted with hexane. $1 \mathrm{~mL}$ final solution was obtained after concentrating the elution solution and transferring the solvent to acetonitrile, analyzed with HPLC [23].

\section{Results and Discussion}

The structures of the ILs, MNPs, and the functional MNPs were confirmed by the FTIR spectra (Figure 1; H-NMR and C-NMR spectra can be obtained from the support information). The peaks in the naked $\mathrm{Fe}_{3} \mathrm{O}_{4}$ and MNPDTIMPF $_{6}$ at $558 \mathrm{~cm}^{-1}$ were due to the Fe-O group and the peaks in DTIMPF $_{6}$ and MNP-DTIMPF 6 at $850 \mathrm{~cm}^{-1}$, $1654 \mathrm{~cm}^{-1}$, and $2931 \mathrm{~cm}^{-1}$ were due to the $-\mathrm{P}-\mathrm{F},-\mathrm{C}=\mathrm{N}-$, and $\mathrm{C}-\mathrm{H}$ groups, respectively. These results demonstrated the successful bonding of DTIMPF $_{6}$ to the naked MNPs by covalent bonds. The peaks in the MNP-DTIMPF 6 at $2360 \mathrm{~cm}^{-1}$ might be due to the $-\mathrm{C}=\mathrm{O}$ of $\mathrm{CO}_{2}$ absorbed on the surface of MNP-DTIMPF 6 .

TEM images showed the naked MNPs (Figure 2(a)) and a representative ionic liquid-modified MNPs (MNPDTIMPF $_{6}$; Figures 2(b) and 2(c)) were quasi-spheres with a mean diameter of about $12 \mathrm{~nm}$. The naked and modified MNPs showed no differences in shape or size. No changes In shape and size of the MNPs were observed after treatment with the water samples. Our modified MNPs differed from coated shell-core structure MNPs, which had a thick coated shell [4-7]. Elemental analysis data showed that the carbon contents of ionic liquid-modified MNPs ranged from 4.25 to $7.15 \%$ depending on the alkyl chain length, whereas the carbon content of naked $\mathrm{Fe}_{3} \mathrm{O}_{4}$ was $0 \%$ (Table 2). The $\mathrm{N}$ and $\mathrm{H}$ contents of naked $\mathrm{Fe}_{3} \mathrm{O}_{4}$ might come from air and water absorbed on the surface of MNPs.

Thermogravimetric analysis (TGA) of naked MNPs detected no significant peaks (Figure 3). There was a $3 \%$ weight loss as the temperature increased from $100^{\circ} \mathrm{C}$ to $700^{\circ} \mathrm{C}$, which might be due to a loss of absorbed water. In contrast, the functionalized MNPs lost weight in two steps. The first step occurred over the range $100-250^{\circ} \mathrm{C}$ and might also be due to the loss of absorbed water. The second step consisted of a weight loss of about $5 \%$ over the range $250-450^{\circ} \mathrm{C}$ and might be due to the burning of bonded ionic liquids. At higher temperatures of $450-700^{\circ} \mathrm{C}$, the weight remained constant, implying the presence of only $\mathrm{Fe}_{3} \mathrm{O}_{4}$ left within the temperature range.

The XRD patterns of naked $\mathrm{Fe}_{3} \mathrm{O}_{4}$ and a representative modified MNP (MNP-DTIMPF ${ }_{6}$; Figure 4) had six characteristic peaks $\left(2 \theta=30.1,35.5,43.1,53.4,57.0\right.$, and $\left.62.6^{\circ}\right)$, corresponding to the Miller indices (220), (311), (400), (422), (511) and (440), respectively. The same peaks were observed for all the modified MNPs. These peaks were in agreement with those in the Mineral Powder Diffraction File Data Book (International Center for Diffraction Data, No. 19629), indicating that the MNPs were pure $\mathrm{Fe}_{3} \mathrm{O}_{4}$ with a 
(1) $2 \mathrm{Fe}^{3+}+\mathrm{Fe}^{2+}+8 \mathrm{OH}^{-} \longrightarrow \mathrm{Fe}_{3} \mathrm{O}_{4}$

(2)<smiles>CCCCCC[As]CCCN1CC[Al](CC[Al]CCC)CC1</smiles>

$(\mathrm{EtO})_{3} \mathrm{Si} \backslash>+\mathrm{Cl}_{\mathrm{Cl}^{+}}^{\mathrm{F}}$<smiles>CCCCCCN1CCN(CCC[AlH2]CCC)C1</smiles>

(3)
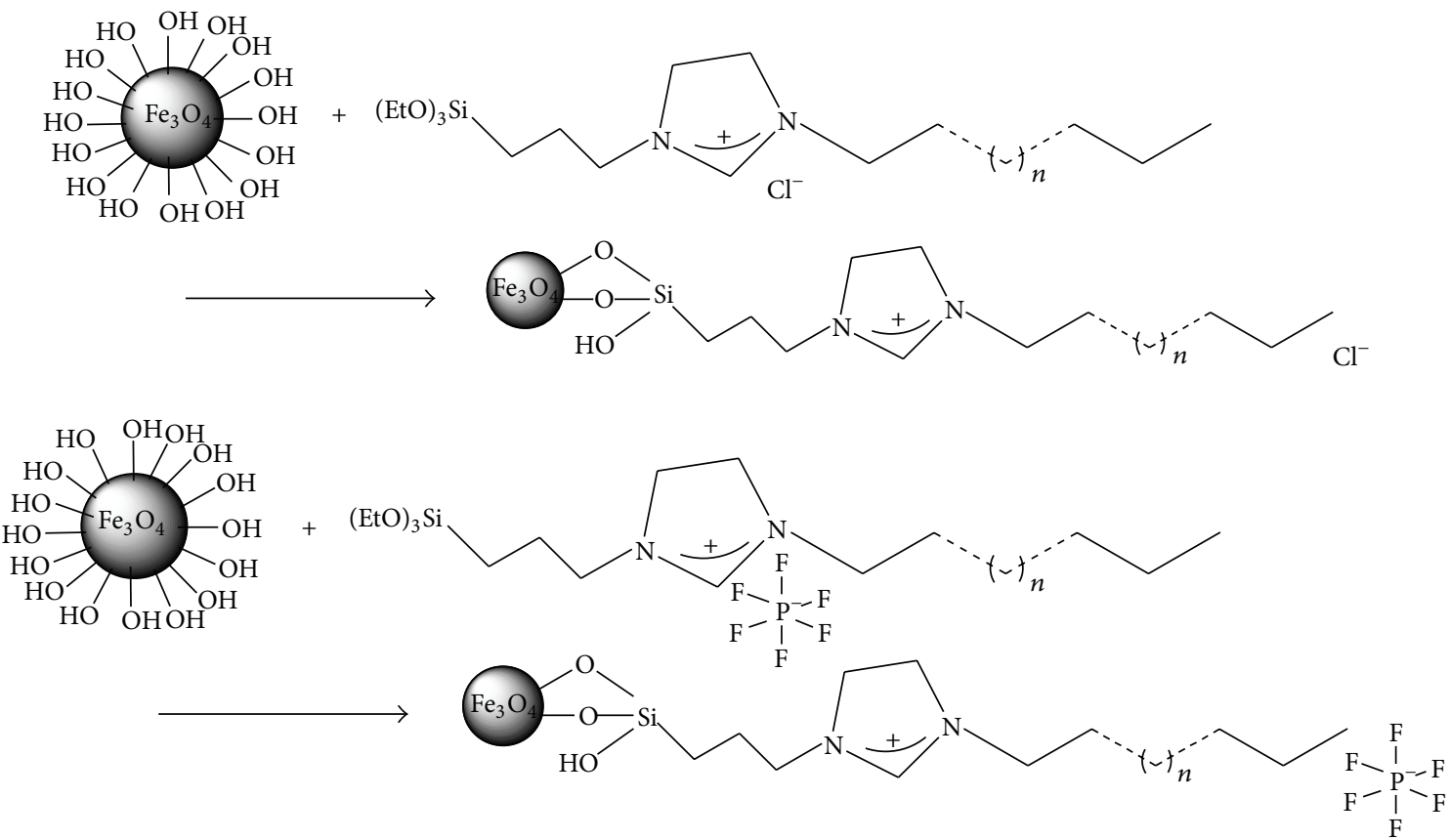

Scheme 1: Synthesis of ionic liquid-modified MNPs (alkyl=hexyl, octyl, and decyl).

spinel structure. Also, the binding process did not result in a phase change of the modified MNPs. Plots of magnetization versus magnetic field ( $\mathrm{M}-\mathrm{H}$ loop) at $25^{\circ} \mathrm{C}$, obtained with a superconducting interference device (SQUID), showed very weak hysteresis in both naked $\mathrm{Fe}_{3} \mathrm{O}_{4}$ and MNP-DTIMPF 6 nanoparticles (Figure 5), indicating that the modified MNPs were superparamagnetic. The saturation magnetization (M) of modified MNPs (60.8 to $63.2 \mathrm{emu} \mathrm{g}^{-1}$ ) was only slightly less than that of naked $\mathrm{Fe}_{3} \mathrm{O}_{4}$ nanoparticles $\left(65.8 \mathrm{emu} \mathrm{g}^{-1}\right)$, indicating that it was little affected by modification of the surface of MNPs. Superparamagnetism of ionic liquid-modified MNPs was an advantage because it prevented the aggregation of MNPs, so that MNPs can be dispersed into a solution again after removal of an external magnet.

The solubility of MNPs strongly depended on the alkyl chain length and anions of the ionic liquid. Modification of MNPs with 1-butyl-3-TIM chloride greatly increased their solubility in water and polar organic solvents [24]. In the 


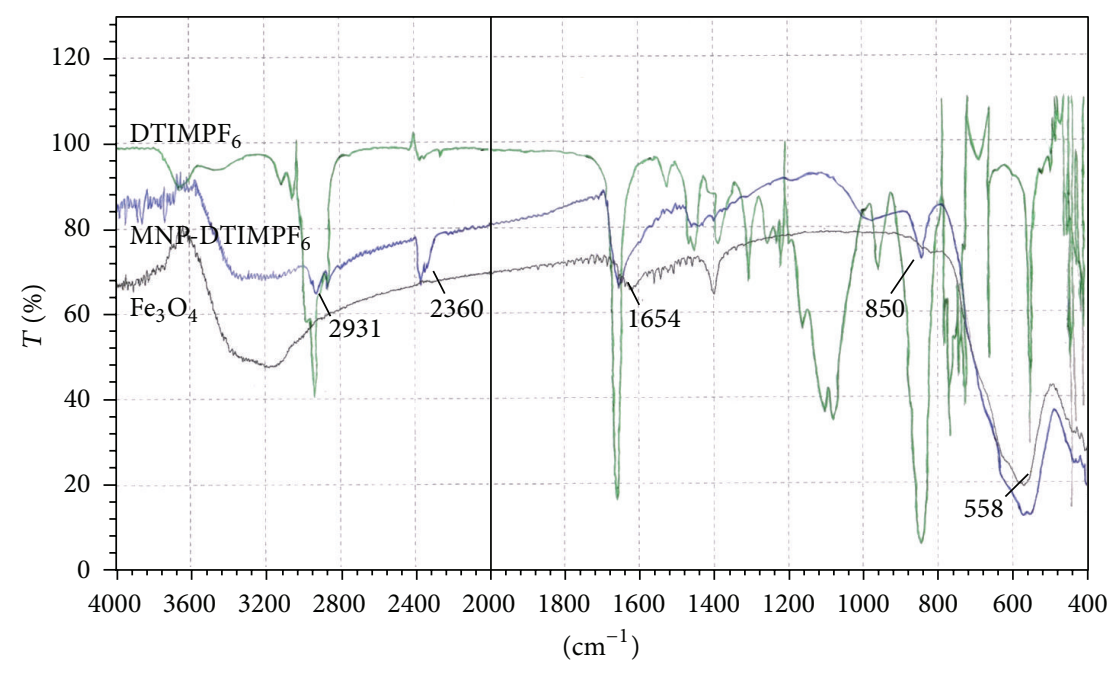

FIGURE 1: IR spectra of $\mathrm{Fe}_{3} \mathrm{O}_{4}, \mathrm{DTIMPF}_{6}$, and MNP-DTIMPF .

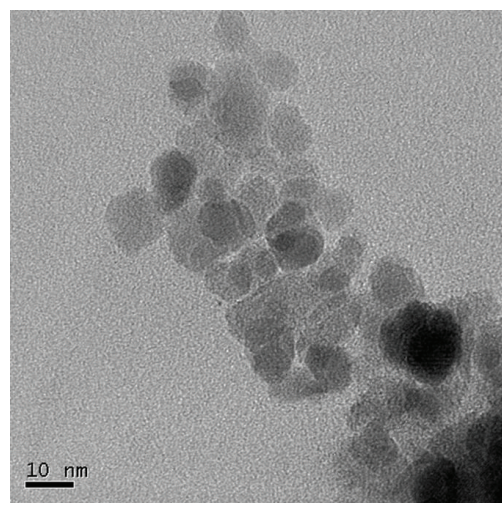

(a)

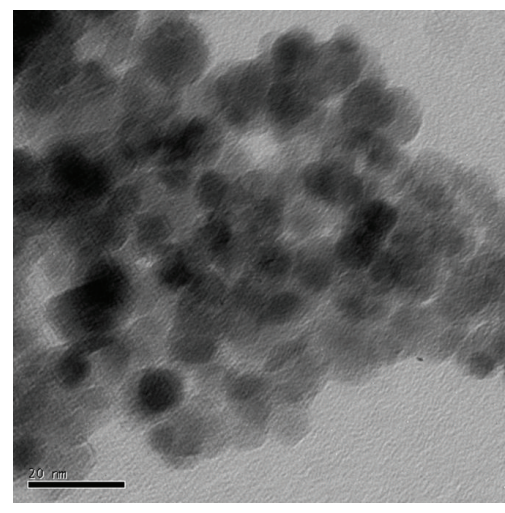

(b)

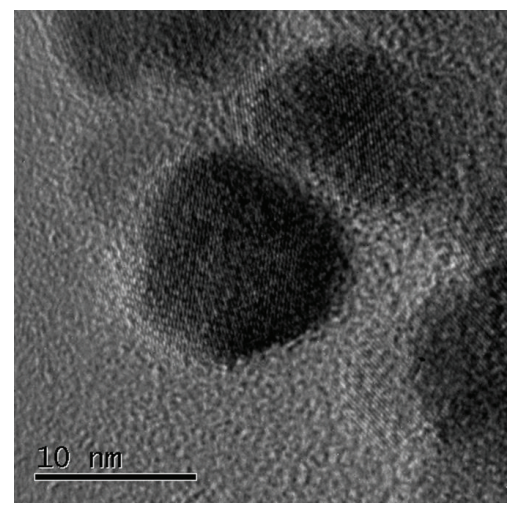

(c)

FIgure 2: (a) TEM of $\mathrm{Fe}_{3} \mathrm{O}_{4}$, (b) TEM of MNP-DTIMPF , and (c) HRTEM of MNP-DTIMPF .

TABLE 1: Ionic liquids synthesized for this study.

\begin{tabular}{|c|c|c|}
\hline Abbrev. & Ionic liquid & $\mathrm{M}\left[\mathrm{g} \mathrm{mol}^{-1}\right]$ \\
\hline HTIMCl & 1-Hexyl-3-TIM chloride* & 504 \\
\hline OTIMCl & 1-Octyl-3-TIM chloride & 532 \\
\hline DTIMCl & 1-Decyl-3-TIM chloride & 560 \\
\hline HTIMPF $_{6}$ & 1-Hexyl-3-TIM hexafluorophosphate & 395 \\
\hline OTIMPF $_{6}$ & 1-Octyl-3-TIM hexafluorophosphate & 423 \\
\hline DTIMPF $_{6}$ & 1-Decyl-3-TIM hexafluorophosphate & 451 \\
\hline
\end{tabular}

*TIM: (3-triethoxysilylpropyl)-4,5-dihydroimidazolium.

present work, the modified MNPs were not soluble in water regardless of the alkyl chain and anions, although they could be dispersed ultrasonically. However, they were soluble and stable in polar organic solvents (e.g., $\mathrm{MeOH}, \mathrm{EtOH}$, and $\mathrm{CH}_{2} \mathrm{Cl}_{2}$ ) to varying degrees depending on the solvent. In strong polar solvents like $\mathrm{MeOH}$ and $\mathrm{EtOH}$, the modified MNPs with chloride as anion were more soluble than those with $\mathrm{PF}_{6}{ }^{-}$as anion. The solubility decreased with increasing alkyl chain length: hexyl > octyl > decyl. On the other hand,
TABLE 2: Results of elemental analysis and TGA of MNPs.

\begin{tabular}{|c|c|c|c|c|}
\hline \multirow{2}{*}{ MNPs } & \multicolumn{3}{|c|}{ Elemental analysis (\%) } & \multirow{2}{*}{$\begin{array}{c}\text { TGA (\%) } \\
\text { A }\end{array}$} \\
\hline & $\mathrm{C}$ & $\mathrm{H}$ & $\mathrm{N}$ & \\
\hline $\mathrm{Fe}_{3} \mathrm{O}_{4}$ & 0.00 & 0.60 & 0.43 & \\
\hline MNP-HTIMPF $_{6}$ & 4.25 & 1.08 & 0.89 & 4.8 \\
\hline MNP-OTIMPF $_{6}$ & 5.42 & 1.28 & 1.33 & 4.5 \\
\hline MNP-DTIMPF $_{6}$ & 6.35 & 1.45 & 0.98 & 6.5 \\
\hline MNP-HTIMCl & 4.54 & 1.10 & 0.99 & 4.8 \\
\hline MNP-OTIMCl & 4.99 & 1.17 & 0.97 & 4.6 \\
\hline MNP-DTIMCl & 7.15 & 1.55 & 1.09 & 8.5 \\
\hline
\end{tabular}

in $\mathrm{CH}_{2} \mathrm{Cl}_{2}$, a weak polar solvent, the modified MNPs with $\mathrm{PF}_{6}{ }^{-}$as anion were more soluble, with solubility increasing with increasing chain length: decyl > octyl > hexyl. Strangely, MNP-OTIMPF $_{6}$ has highest solubility in both strong and weak polar solvents (Figure 6). Its unexpected high solubility in organic solvent might be related in some way to its low melting point (OTIMPF$_{6}$ was orange-colored liquid while 


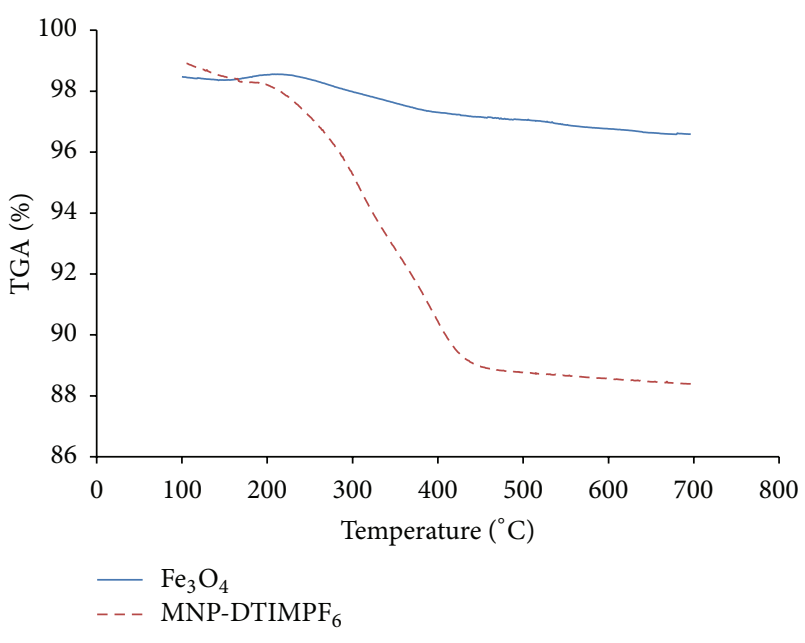

Figure 3: TGA of $\mathrm{Fe}_{3} \mathrm{O}_{4}$ and MNP-DTIMPF $F_{6}$.

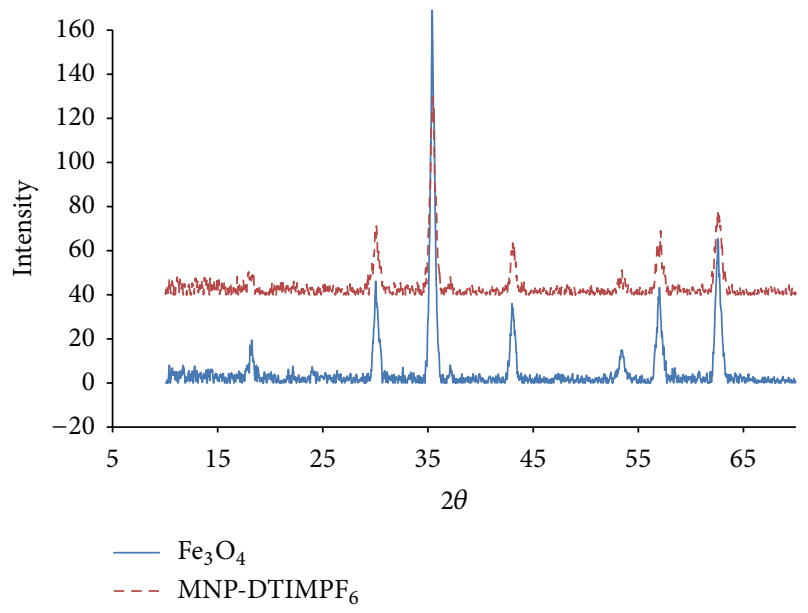

FIGURE 4: X-ray diffraction patterns of $\mathrm{Fe}_{3} \mathrm{O}_{4}$ and MNP-DTIMPF 6 (Miller indices of the peaks are indicated).

HTIMPF $_{6}$ and DTIMPF$_{6}$ were colorless powder). Neither naked MNP nor modified MNPs were soluble in nonpolar organic solvents, such as hexane.

PAHs were designated as the priority monitoring pollutants due to their potential carcinogenic or mutagenic properties. In the present work, ten PAHs with $4 \sim 6$ fused rings (fluoranthene (Flu), pyrene (Pyr), benz[a] anthracene (BaA), chrysene (Chr), benzo[b]fluoranthrene (BbF), benzo $[k]$ fluoranthrene $(\mathrm{BkF})$, benzo[a]pyrene $(\mathrm{BaP})$, dibenz $[a, h]$ anthracene (DBA), benzo[ $g, h, i]$ perylene (BgPe), and indeno $[1,2,3-c d]$ pyrene (IDP), were selected as target to examine the functionality of the MNPs. The results of the spiked river water samples with PAHs standards were compared with the original water samples. Recoveries of PAHs from spiked water samples with ionic liquid-modified MNPs were much higher than those of the naked $\mathrm{Fe}_{3} \mathrm{O}_{4}$, clearly demonstrating the extraction ability of imidazolium-based ionic liquids of the modified MNPs to PAHs. Ionic liquid-modified MNPs with hexafluorophosphate anion had much higher recoveries than MNPs with chloride anion when the alkyl was hexyl, while

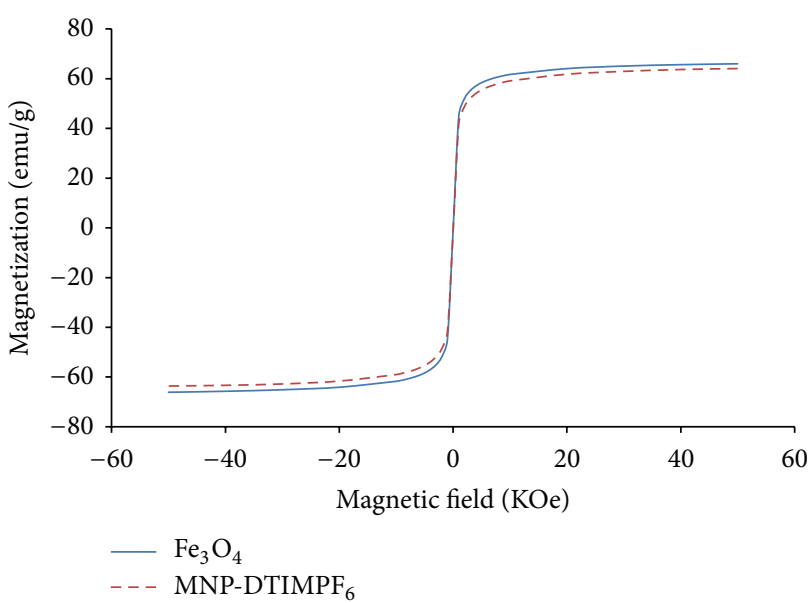

FIGURE 5: Magnetization versus magnetic field for $\mathrm{Fe}_{3} \mathrm{O}_{4}$ and MNP$\mathrm{DTIMPF}_{6}$ at $25^{\circ} \mathrm{C}$.

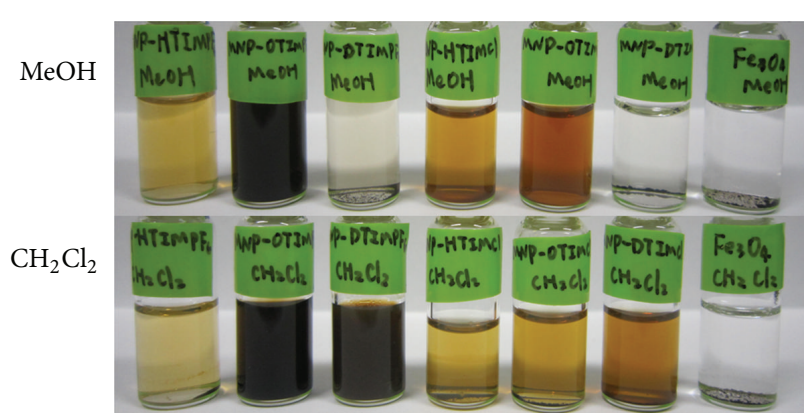

FIGURE 6: Solubility of the naked and modified MNPs in methanol and $\mathrm{CH}_{2} \mathrm{Cl}_{2}$.

they had similar recoveries when the alkyl was octyl or decyl. In general, recoveries of PAHs were higher for MNPs with $\mathrm{PF}_{6}{ }^{-}$anion than that with $\mathrm{Cl}^{-}$anion. $\pi-\pi$ and hydrophobic interactions between PAHs and N, ${ }^{\prime}$-dialkylimidazoliumbased ionic liquids were presented [19]. The main interaction should be $\pi-\pi$ interaction when the alkyl is hexyl. The anions greatly affected the property of $\mathrm{N}, \mathrm{N}^{\prime}$-dialkylimidazoliumbased ionic liquid. However, the property of ionic liquid gradually depended on the cation with increasing of alkyl chain length and hydrophobic interaction played an important role to preconcentration. Hence, a better detection efficiency might be improved if MNPs were modified with ionic liquids with longer alkyl chain, for example, octadecyl, and $\mathrm{PF}_{6}{ }^{-}$or more hydrophobic anions were provided. As a demonstration, $\mathrm{MNP}_{-\mathrm{DTIMPF}}$ was found to efficiently preconcentrate several PAHs (Flu, Pyr, BaA, Chr, BbF, BkF, and BaP) from a river water sample (Figure 7). A similar chromatogram was obtained with the river water sample spiked with $\mathrm{PAH}$ standard solutions, confirming the detections.

\section{Conclusion}

1-Alkyl-3-(3-triethoxysilylpropyl)-4,5-dihydroimidazolium anion ionic liquids were synthesized and successfully bonded to the MNPs. Measurement of IR, NMR, TGA, TEM, and 


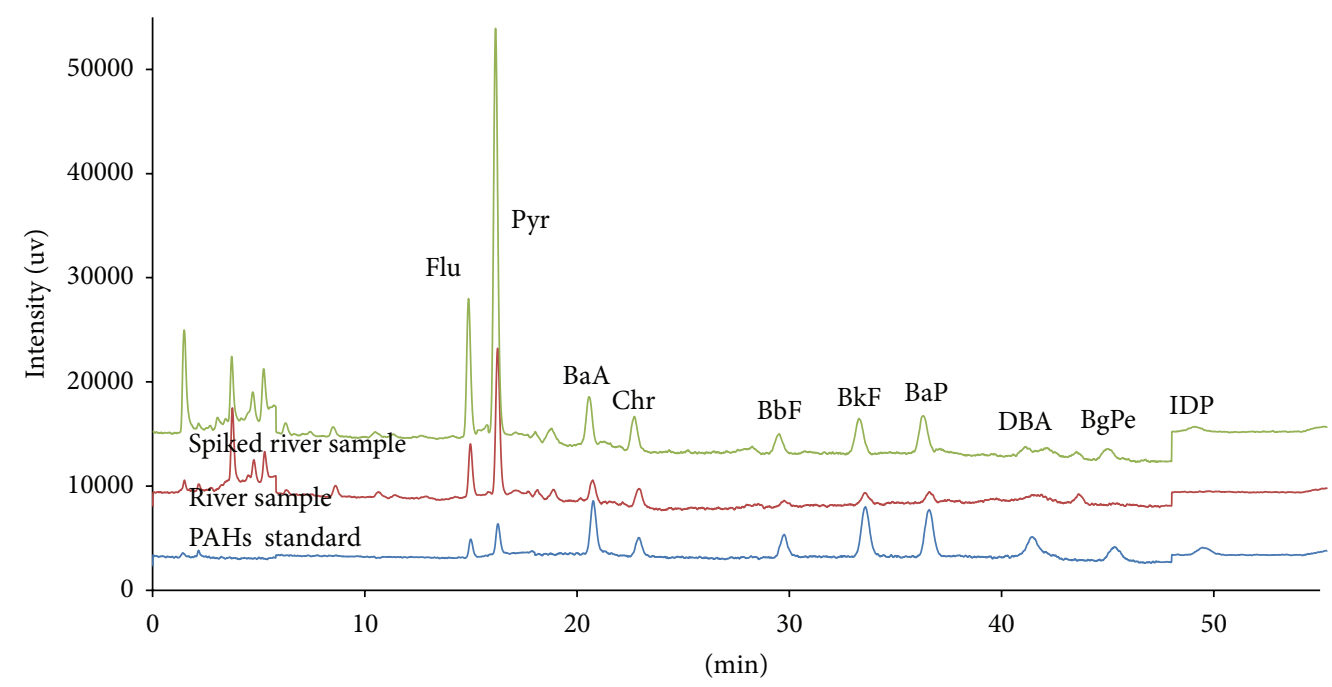

FIGURE 7: Chromatograms of PAHs standard, river water sample, and spiked river water sample.

elemental analysis confirmed the structures. XRD and MPMS analysis illustrated the $\mathrm{Fe}_{3} \mathrm{O}_{4}$ core of the MNPs with superparamagnetism. Functionality of MNPs with ionic liquids greatly influenced their solubility in organic solvents and facilitated the specific extraction capability to organic pollutants.

\section{Acknowledgments}

This work was supported in part by a Grant-in-Aid for scientific research from the Ministry of Education, Science, Sport and Culture and by funds from the Ministry of Environment, Japan. The authors thank Professor M. Okuno for XRD measurement and Professor T. Yamagishi for his help with TGA analysis. Y. Li was supported by a Postdoctoral Fellowship for Foreign Researchers from the Japan Society for the Promotion of Sciences (JSPS).

\section{References}

[1] R. Hao, R. Xing, Z. Xu, Y. Hou, S. Goo, and S. Sun, "Synthesis, functionalization, and biomedical applications of multifunctional magnetic nanoparticles," Advanced Materials, vol. 22, no. 25, pp. 2729-2742, 2010.

[2] M. Safarikova, P. Lunackova, K. Komarek, T. Hubka, and I. Safarik, "Preconcentration of middle oxyethylated nonylphenols from water samples on magnetic solid phase," Journal of Magnetism and Magnetic Materials, vol. 311, no. 1, pp. 405-408, 2007.

[3] C. $\mathrm{Xu}, \mathrm{K} . \mathrm{Xu}, \mathrm{H} . \mathrm{Gu}$ et al., "Dopamine as a robust anchor to immobilize functional molecules on the iron oxide shell of magnetic nanoparticles," Journal of the American Chemical Society, vol. 126, no. 32, pp. 9938-9939, 2004.

[4] R. D. Palma, S. Peeters, M. J. V. Bael et al., "Silane ligand exchange to make hydrophobic superparamagnetic nanoparticles water-dispersible," Chemistry of Materials, vol. 19, no. 7, pp. 1821-1831, 2007.

[5] S. X. Zhang, H. Y. Niu, Z. J. Hu, Y. Q. Cai, and Y. L. Shi, "Preparation of carbon coated $\mathrm{Fe}_{3} \mathrm{O}_{4}$ nanoparticles and their application for solid-phase extraction of polycyclic aromatic hydrocarbons from environmental water samples," Journal of Chromatography A, vol. 1217, no. 29, pp. 4757-4764, 2010.

[6] L. Sun, C. Z. Zhang, L. Chen et al., "Preparation of aluminacoated magnetite nanoparticle for extraction of trimethoprim from environmental water samples based on mixed hemimicelles solid-phase extraction," Analytica Chimica Acta, vol. 638, no. 2, pp. 162-168, 2009.

[7] J. S. Suleiman, B. Hu, H. Y. Peng, and C. Z. Huang, "Separation/preconcentration of trace amounts of $\mathrm{Cr}, \mathrm{Cu}$ and $\mathrm{Pb}$ in environmental samples by magnetic solid-phase extraction with Bismuthiol-II-immobilized magnetic nanoparticles and their determination by ICP-OES," Talanta, vol. 77, no. 5, pp. 1579-1583, 2009.

[8] A. Ballesteros-Gómez and S. Rubio, "Hemimicelles of alkyl carboxylates chemisorbed onto magnetic nanoparticles: Study and application to the extraction of carcinogenic polycyclic aromatic hydrocarbons in environmental water samples," Analytical Chemistry, vol. 81, no. 21, pp. 9012-9020, 2009.

[9] M. Smiglak, A. Metlen, and R. D. Rogers, “The second evolution of ionic liquids: from solvents and separations to advanced materials-energetic examples from the ionic liquid cookbook," Accounts of Chemical Research, vol. 40, no. 11, pp. 1182-1192, 2007.

[10] M. Tobiszewski, A. Mechlińska, B. Zygmunt, and J. Namieśnik, "Green analytical chemistry in sample preparation for determination of trace organic pollutants," Trends in Analytical Chemistry, vol. 28, no. 8, pp. 943-951, 2009.

[11] K. Yamaguchi, C. Yoshida, S. Uchida, and N. Mizuno, "Peroxotungstate immobilized on ionic liquid-modified silica as a heterogeneous epoxidation catalyst with hydrogen peroxide," Journal of the American Chemical Society, vol. 127, no. 2, pp. 530531, 2005.

[12] S. Shylesh, V. Schünemann, and W. R. Thiel, "Magnetically separable nanocatalysts: bridges between homogeneous and heterogeneous catalysis," Angewandte Chemie-International Edition, vol. 49, no. 20, pp. 3428-3459, 2010.

[13] T. Torimoto, T. Tsuda, K. I. Okazaki, and S. Kuwabata, "New frontiers in materials science opened by ionic liquids," Advanced Materials, vol. 22, no. 11, pp. 1196-1221, 2010. 
[14] X. X. Han and D. W. Armstrong, "Ionic liquids in separations," Accounts of Chemical Research, vol. 40, no. 11, pp. 1079-1086, 2007.

[15] S. G. Lee, "Functionalized imidazolium salts for task-specific ionic liquids and their applications," Chemical Communication, no. 10, pp. 1049-1063, 2006.

[16] X. J. Sun, Y. L. Zhu, P. Wang, J. Li, C. Y. Wu, and J. Xing, "High temperature and highly selective stationary phases of ionic liquid bonded polysiloxanes for gas chromatography," Journal of Chromatography A, vol. 1218, no. 6, pp. 833-841, 2011.

[17] W. Y. Zhao, M. Han, S. G. Dai, J. Xu, and P. Wang, "Ionic liquidcontaining semipermeable membrane devices for monitoring the polycyclic aromatic hydrocarbons in water," Chemosphere, vol. 62, no. 10, pp. 1623-1629, 2006.

[18] K. P. Huang, G. R. Wang, B. Y. Huang, and C. Y. Liu, "Preparation and application of ionic liquid-coated fused-silica capillary fibers for solid-phase microextraction," Analytica Chimica Acta, vol. 645, no. 1-2, pp. 42-47, 2009.

[19] H. D. Qiu, M. Takafuji, X. Liu, S. X. Jiang, and H. Ihara, "Investigation of $\pi-\pi$ and ion-dipole interactions on 1-allyl-3butylimidazolium ionic liquid-modified silica stationary phase in reversed-phase liquid chromatography," Journal of Chromatography A, vol. 1217, no. 32, pp. 5190-5196, 2010.

[20] C. Yao, W. R. Pitner, and J. L. Anderson, "Ionic liquids containing the tris(pentafluoroethyl)trifluorophosphate anion: a new class of highly selective and ultra hydrophobic solvents for the extraction of polycyclic aromatic hydrocarbons using single drop microextraction," Analytical Chemistry, vol. 81, no. 12, pp. 5054-5063, 2009.

[21] R. J. Soukup-Hein, M. M. Warnke, and D. W. Armstrong, "Ionic liquids in analytical chemistry," Annual Review of Analytical Chemistry, vol. 2, pp. 145-168, 2009.

[22] R. Massart, "Preparation of aqueous magnetic liquid in alkaline and acidic media," IEEE Transactions on Magnetics, vol. 17, no. 2, pp. 1247-1248, 1981.

[23] Y. Li, S. Yoshida, Y. Chondo et al., "On-line concentration and fluorescence determination HPLC for polycyclic aromatic hydrocarbons in seawater samples and its application to Japan Sea," Chemical and Pharmaceutical Bulletin, vol. 64, no. 4, pp. 531-535, 2012.

[24] R. Abu-Reziq, D. S. Wang, M. Post, and H. Alper, "Platinum nanoparticles supported on ionic liquid-modified magnetic nanoparticles: selective hydrogenation catalysts," Advanced Synthesis and Catalysis, vol. 349, no. 13, pp. 2145-2150, 2007. 

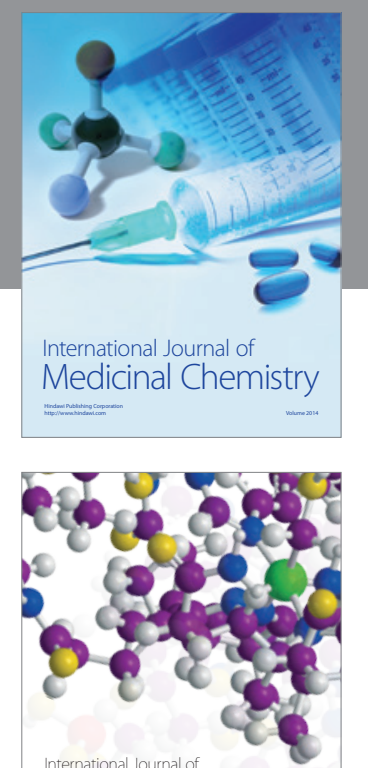

\section{Carbohydrate} Chemistry

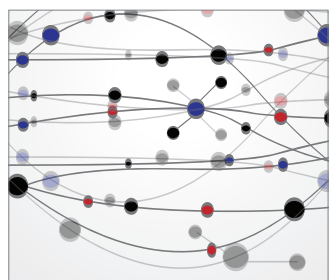

The Scientific World Journal
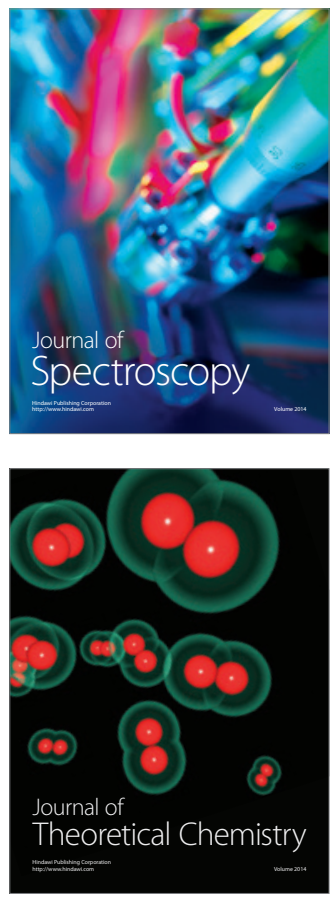
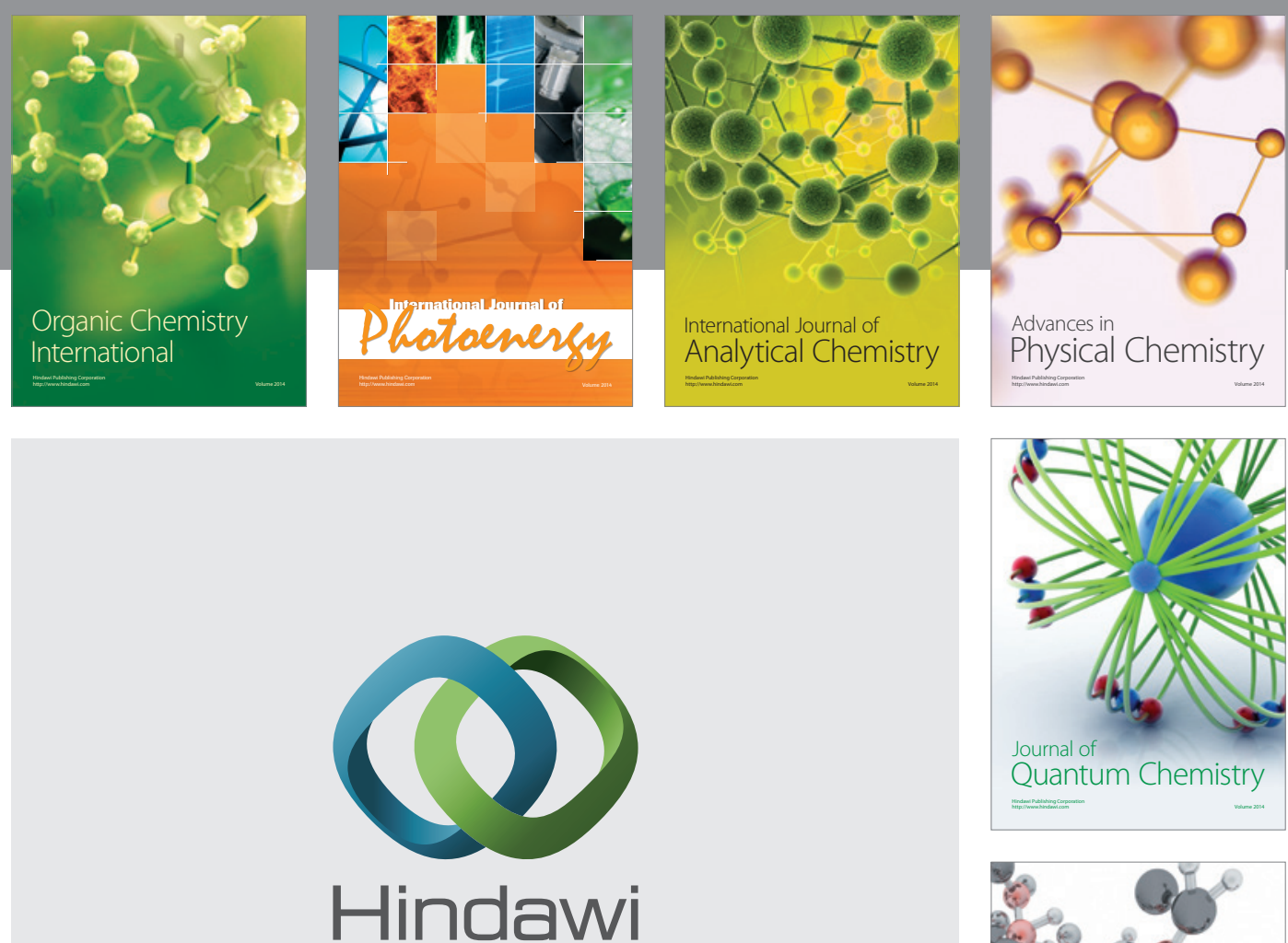

Submit your manuscripts at

http://www.hindawi.com

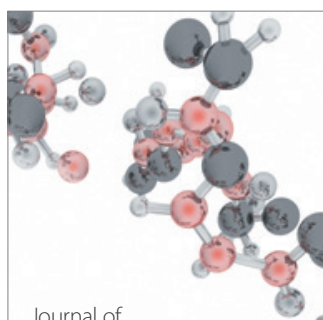

Analytical Methods

in Chemistry

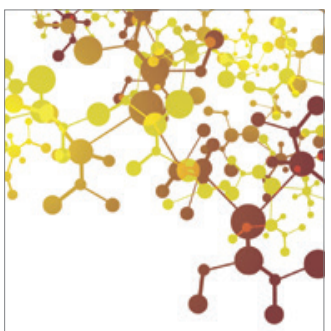

Journal of

Applied Chemistry

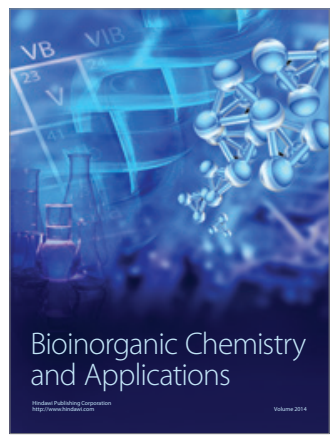

Inorganic Chemistry
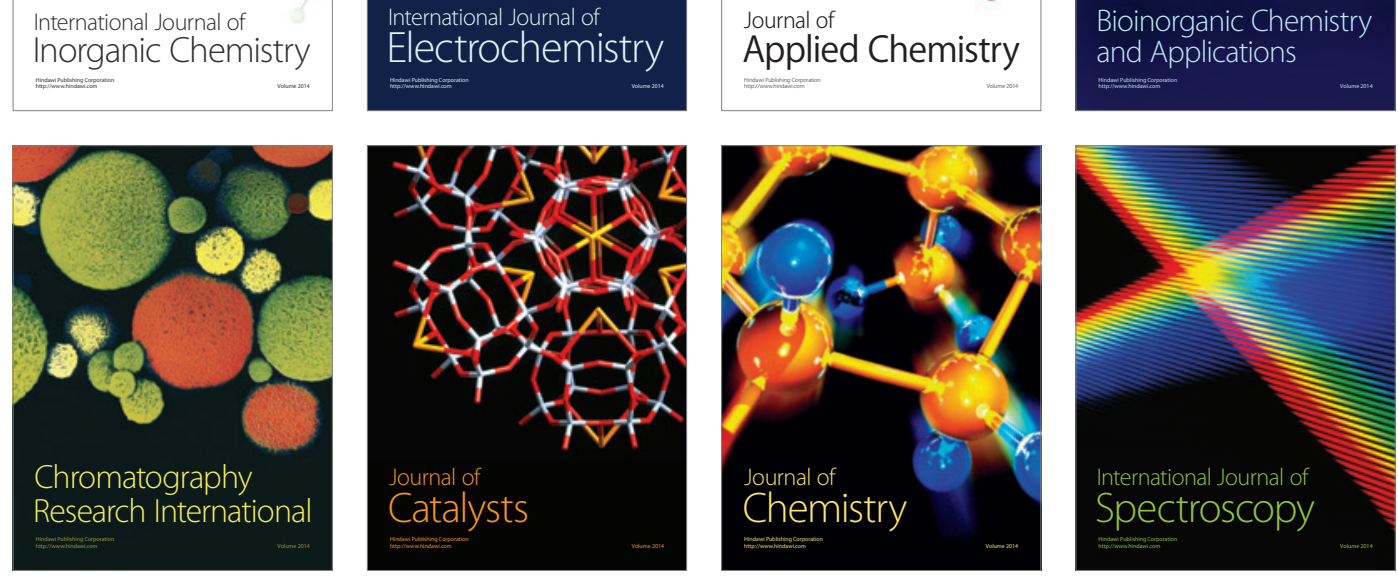